\title{
Medication-related osteonecrosis of the jaw
}

\author{
Héliton Spindola Antunes ${ }^{1,2}$ Rogerio Jardim Caldas, ${ }^{1,3,4}$ Fabiana Caramori Noal Granzotto, ${ }^{1,5}$ Geisa Badauy Lauria da Silva, ${ }^{1,6}$ Walmir Mello, ${ }^{1,7}$ Paulo Sérgio \\ da Silva Santos ${ }^{1,3}$ \\ 'Dental Committee of Brazilian Society of Hematology, Hemotherapy and Cell Therapy (ABHH), São Paulo, SP, Brazil \\ ${ }^{2}$ Clinical Research Division, Brazilian National Cancer Institute (INCA), Rio de Janeiro, RJ, Brazil \\ ${ }^{3}$ Department of Surgery, Stomatology, Pathology and Radiology, Bauru School of Dentistry, University of São Paulo, Bauru, SP, Brazil \\ ${ }^{4}$ Dentistry Section, Mario Kroeff Hospital, Rio de Janeiro, RJ, Brazil \\ ${ }^{5}$ Dentistry Section, Regina Hospital, Novo Hamburgo, RS, Brazil \\ ${ }^{6}$ Hematopoietic Stem Cell Transplant Unit, Araujo Jorge Hospital, Goiânia, GO, Brazil \\ ${ }^{7}$ Dentistry Section, Samaritano Hospital, São Paulo, SP, Brazil \\ - Conflicts of interest: none declared.
}

\section{Dear editor,}

$\mathrm{T}$ This is a short review about the medication-related osteonecrosis of the jaw (MRONJ) and clinical implications. MRONJ is defined by the combination of the following signs: 1. Current or previous use of antiresorptive or antiangiogenic drugs; 2 . Bone exposure of the jaws or extraoral fistula persisting for more than 8 weeks; 3 . Lack of history of radiotherapy in the maxillofacial region. ${ }^{1,2}$

The incidence of MRONJ is variable. This condition affects 1.04 to 1.69 per 100.000 patients/year for individuals receiving oral bisphosphonates (BPs). For oncology patients under treatment with intravenous BPs or even with denosumab, the incidence ranges from 0 to 12.222 and from 0 to 2.316 per 100.000 patients/year respectively. ${ }^{3-8}$ Considering the duration of treatment, the incidence varies from 0 to $0.21 \%$ in individuals who have used oral BPs for more than 4 years. MRONJ is also 50-100 times more frequent among patients with cancer and bone metastases compared to those suffering from osteoporosis. ${ }^{2}$

Despite many clinical and laboratory studies, the exact mechanism by which osteonecrosis occurs is still under investigation. However, some evidences of its pathophysiology are established as follows: suppression of bone remodeling; infection and inflammation; inhibition of angiogenesis; soft tissue toxicity; immunity-related theories; bone microfractures; and local factors (dental extractions; anatomical alterations where the oral mucosa becomes thin and more susceptible to traumas; and oral diseases, such as periodontal and periapical affections, and dentoalveolar abscesses). ${ }^{1,2}$

MRONJ has been mainly associated with the administration of BPs and anti-receptor activator nuclear factor kappa-B ligand (anti-RANKL) monoclonal antibody, such as denosumab ${ }^{9}$, as a result of bone turnover suppression. The nitrogen-containing BPs (pamidronate, ibandronate, zoledronate, risedronate, alendronate) inhibit the activity of farnesyl diphosphate synthase, which is a key enzyme in the mevalonate pathway. Consequently, a deficiency of geranyl diphosphate and farnesyl diphosphate arises inside the cells, resulting not only in inhibition of mature osteoclasts but also preventing osteoclast precursors from differentiating and migrating towards inflammatory osteolytic lesions. ${ }^{9-13}$ Denosumab is a fully humanized antibody against RANK ligand (RANKL), also capable of potently inhibiting osteoclast-mediate bone resorption. ${ }^{9}$ Novel antiangiogenic medications (tyrosine kinase inhibitors and monoclonal antibody-targeting VEFG) such as bevacizumab, sunitinib, sorafenib and sirolimus were added to the list of drugs related to necrosis of the jaws. Angiogenesis inhibitors interfere with the formation of new vessels by binding to signaling molecules, causing a disruption of the angiogenesis cascade. ${ }^{14}$ Antiangiogenic effects have been reported in studies involving cancer patients treated with zoledronic acid. Decreased blood levels of vascular endothelial growth factor (VEGF) have been demonstrated in this population. ${ }^{15}$

Recently, the clinical presentation without bone exposure integrates a new aspect of the disease, corresponding to stage 0 . At this stage, only unspecific signs and symptoms are noted, such as pain and bone sclerosis. Radiographic changes were thus included as diagnostic criteria for the disease. The subsequent stages consist of exposed and necrotic bone or fistulas that probes to bone without infection (stage 1) or associated with it (stage 2). It may be worsened by pathological fracture or extraoral fistula (stage 3). ${ }^{1}$

For the management of MRONJ, non-surgical and surgical approaches are considered in clinical practice. Non-surgical treatments aim at pain and infection control, including oral hygiene care (medical care and home care), eradication of periodontal disease, use of antimicrobial mouthwashes (chlorhexidine gluconate $0.12 \%$ ), antibiotic therapy and systemic corticosteroid therapy. ${ }^{3}$ Surgical treatment consists of debridement and removal of infected or necrotic bone sequestration. In severe cases with extensive lesions, resection 
of bone segments may be indicated. ${ }^{16}$

It is important to consider that the risk of developing MRONJ is real, despite its low incidence. In addition, MRONJ at any stage may deeply impair oral functions and ultimately complicate cancer treatment, nutrition, and den- tal care. Therefore, surgical interventions involving bone manipulation in patients taking antiresorptive or antiangiogenic drugs should consider the eligibility of the procedure based on scientific evidence.

\section{References}

1. Ruggiero SL, Dodson TB, Fantasia J, Goodday R, Aghaloo T, Mehrotra B, et al. American Association of Oral and Maxillofacial Surgeons position paper on medication-related osteonecrosis of the jaw-2014 update. J Oral Maxillofac Surg. 2014;72(10):1938-56.

2. Kim KM, Rhee Y, Kwon YD, Kwon TG, Lee JK, Kim DY. Medication Related Osteonecrosis of the Jaw: 2015 Position Statement of the Korean Society for Bone and Mineral Research and the Korean Association of Oral and Maxillofacial Surgeons. J Bone Metab. 2015;22(4):151-65.

3. Khan AA, Morrison A, Hanley DA, Felsenberg D, McCauley LK, O'Ryan F, et al. Diagnosis and management of osteonecrosis of the jaw: a systematic review and international consensus. J Bone Miner Res. 2015;30(1):3-23.

4. Ulmner M, Jarnbring F, Törring O. Osteonecrosis of the jaw in Sweden associated with the oral use of bisphosphonate. J Oral Maxillofac Surg. 2014;72(1):76-82. 5. Etminan M, Aminzadeh K, Matthew IR, Brophy JM. Use of oral bisphosphonates and the risk of aseptic osteonecrosis: a nested case-control study. J Rheumatol. 2008;35(4):691-5.

6. Lyles KW, Colón-Emeric CS, Magaziner JS, Adachi JD, Pieper CF, Mautalen $\mathrm{C}$, et al. Zoledronic acid and clinical fractures and mortality after hip fracture. $\mathrm{N}$ Engl J Med. 2007;357(1):1799-809.

7. Tennis P, Rothman KJ, Bohn RL, Tan H, Zavras A, Laskarides C, et al. Incidence of osteonecrosis of the jaw among users of bisphosphonates with selected cancers or osteoporosis. Pharmacoepidemiol Drug Saf. 2012;21(8):810-7.

8. Malden N, Lopes V. An epidemiological study of alendronate-related osteo- necrosis of the jaws. A case series from the south-east of Scotland with attention given to case definition and prevalence. J Bone Miner Metab. 2012;30(2):171-82. 9. Uyanne J, Calhoun CC, Le AD. Antiresorptive drugs-related osteonecrosis of the jaw. Dent Clin N Am. 2014;58(2):369-84.

10-Rogers MJ, Gordon S, Benford HL, Coxon FP, Luckman SP, Monkkonen J, et al. Celular and molecular mechanisms of action of bisphosphonates. Cancer. 2000;88(12 Suppl):2961-78.

11. Reszka AA, Rodan GA. Bisphosphonate mechanism of action. Curr Rheumatol Rep. 2003;5:65-74.

12-Kimachi K, Kajiya H, Nakayama S, Ikebe T, Okabe K. Zoledronic acid inhibits RSNK expression and migration of osteoclast precursors during osteoclastogenesis. Naunyn Schimiedebergs Arch Pharmacol. 2011;383(3):297-308.

13. Lim SS, Lee B, Kim IS, Hwang SJ. Differential modulation of zoledronate and etidronate in osseous healing of an extracted socket and tibia defect. Oral Surg Oral Med Oral Pathol Oral Radiol. 2017;123(1):8-19.

14. Ruggiero SL. Diagnosis and staging of medication-related osteonecrosis of the jaw. Oral Maxillofac Surg Clin N Am. 2015;27(4):479-87.

15. Santini D, Vincenzi B, Dicuonzo G, Avvisati G, Massacesi C, Battistoni F, et al. Zoledronic acid induces significant and long-lasting modifications of circulating angiogenic factors in cancer patients. Clin Cancer Res. 2003;9(8):2893-7.

16. Ferrari S, Bianchi B, Savi A, Poli T, Multino A, Balestreri A, et al. Fibula free flap with endosseous implants for reconstructing a resected mandible in bisphosphonate osteonecrosis. J Oral Maxillofac Surg. 2008;66(5):999-1003.

\section{Mini Curriculum and Author's Contribution}

1. Héliton Spindola Antunes - DDS and PhD. Contribution: manuscript writing, manuscript review, work supervisor and paper submission.

2. Rogerio Jardim Caldas - DDS and MSc. Contribution: manuscript writing, manuscript review.

3. Fabiana Caramori Noal Granzotto - DDS. Contribution: manuscript writing.

4. Geisa Badauy Lauria da Silva - DDS and PhD. Contribution: manuscript writing.

5. Walmir Mello - DDS and MSc. Contribution: manuscript writing.

6. Paulo Sérgio da Silva Santos - DDS and PhD. Contribution: manuscript writing, manuscript review, work supervisor.

Submitted: 02/05/2018 / Accepted for publication: 02/20/2018

Corresponding Author

Héliton Spindola Antunes

E-mail: hspindola@inca.gov.br 